\title{
VALIDACIÓN DE LA ESCALA DE INVOLUCRAMIENTO RELIGIOSO UNIVERSAL AGE I-E 12 EN ESCOLARES ENTRE 8 Y 19 AÑOS DEL NORTE DE CHILE
}

\author{
VALIDATION OF THE AGE UNIVERSAL I-E 12 RELIGIOUS INVOLVEMENT SCALE \\ IN SCHOOLCHILDREN BETWEEN 8 AND 19 YEARS OLD IN NORTHERN CHILE
}

\author{
Jerome Flores Jara* \\ Centro de Justicia Educacional y Escuela de Psicología y Filosofía, Universidad de Tarapacá, \\ Arica - Chile. \\ Alejandra Caqueo-Urízar \\ Centro de Justicia Educacional e Instituto de Alta Investigación, Universidad de Tarapacá, \\ Arica - Chile.

\section{Pascal Hernández} \\ Centro de Justicia Educacional y Escuela de Psicología y Filosofía, Universidad de Tarapacá, \\ Arica - Chile.

\section{Fernanda Vargas} \\ Centro de Justicia Educacional y Escuela de Psicología y Filosofía, Universidad de Tarapacá, \\ Arica - Chile.
}

Recibido septiembre de 2019Received September, 2019

Aceptado diciembre de 2019/Accepted December, 2019

\begin{abstract}
RESUMEN
Antecedentes: La escala Age Universal ha sido ampliamente utilizada como parte de las versiones derivadas de la escala original de involucramiento religioso de Allport y Ros (1967) en población adolescente y adulta. No obstante, no ha sido validada exhaustivamente y en Latinoamérica no cuenta con ningún tipo de validación en población infanto-juvenil. Es relevante contar con una escala que permita medir este constructo en población infanto-juvenil dada la evidencia favorable de involucramiento religiosos sobre variables de salud mental y bienestar en esta población. Objetivo: validar la escala Age-Universal I-E 12 en población infanto-juvenil en Chile. Método: Se realiza un estudio trasversal para para comprobar las propiedades psicométricas de la escala en escolares entre 8 y 19 años del norte de Chile. Se utiliza una muestra de 3857 estudiantes de ambos sexos, que incluye colegios de diferente dependencia. Los datos se analizan mediante ESEM y CFA. Resultados: A diferencia de la dimensionalidad frecuentemente encontrada en universitarios, de tres factores, en el presente estudio se obtiene una estructura bifactorial donde se combina la dimensión intrínseca con la extrínseca personal. Los indicadores de bondad de ajuste son adecuados $(\mathrm{RMSEA}=, 062 ; \mathrm{CFI}=.987 ; \mathrm{TLI}=.984)$. La fiabilidad de la escala total es .91 , en tanto que su dimensión intrínseca combinada es de .91 y su dimensión extrínseca social es de .81. siendo adecuada. Conclusión: La escala cuenta con propiedades psicométricas adecuadas para ser utilizada en Chile y probablemente en Latinoamérica.
\end{abstract}

Palabras Clave: Universal age, Validación, Involucramiento religioso, Niños y adolescentes.

* Autor correspondiente / Corresponding author: jflores@uta.cl 


\begin{abstract}
Background: The Universal Age scale has been widely used as part of versions derived from the original Allport and Ros (1967) scale of religious involvement in adolescent and adult populations. However, it has not been validated exhaustively and in Latin America it does not have any type of validation in child-youth population. It is relevant to have a scale that allows to measure this construct in infantile-juvenile population given the favorable evidence of religious involvement on mental health and well-being variables in this population. Objective: to validate the scale Age-Universal I-E 12 in infantile-juvenile population in Chile. Method: A cross-sectional study is carried out to verify the psychometric properties of the scale in schoolchildren between the ages of 8 and 19 in northern Chile. A sample of 3857 students of both sexes is used, which includes schools of different dependence. The data are analysed by ESEM and CFA. Results: Unlike the dimensionality frequently found in university students, of three factors, in the present study a bifactorial structure is obtained where the intrinsic dimension is combined with the personal extrinsic one. Goodness-of-fit indicators are adequate $(\mathrm{RMSEA}=, 062 ; \mathrm{CFI}=.987$; TLI $=.984)$. The reliability of the full scale is .91 , while its combined intrinsic dimension is .91 and its social extrinsic dimension is .81. being appropriate. Conclusion: The scale has psychometric properties suitable for use in Chile and probably Latin America.
\end{abstract}

Key Words: Universal age, Validation, Religious involvement, Children and adolescents.

\section{INTRODUCCIÓN}

El papel de la religión ha sido debatido ampliamente en la historia del ser humano (Koenig, 2018). Si bien algunos consideran el involucramiento religioso como fuente de patología, otros lo consideran como un factor protector en varios aspectos de la vida, siendo esto último lo que más respaldo ha recibido en la investigación (Burke, Olphen, Eliason, Howell, \& Gonzalez, 2014; Hackney \& Sanders, 2003; Koenig, 2015).

La utilización de instrumentos estandarizados es un requisito esencial para poder generar avances adecuados en la ciencia, puesto que permite comparar apropiadamente resultados entre diferentes investigaciones. Pese a ello, existen varios estudios que han utilizado escalas $a d-h o c$ cuando tienen como objetivo medir involucramiento religioso, ítems extraídos de escalas o mezclas de ítems de diferentes escalas (Chiswick \& Mirtcheva, 2013; Cotton, McGrady, \& Rosenthal, 2010; Hardy et al., 2013; Kim, Harty, Takahashi, \& Voisin, 2017). Otros autores han utilizado medidas ad-hoc de asistencia a servicios religiosos y/o frecuencia de rezo/meditación como medida de involucramiento, lo que tampoco permite precisión en la medición del constructo (Chen \& VanderWeele, 2018).

Abarcar todos los aspectos de la religiosidad es sin duda complejo. Koenig (2018), al revisar la medición de los aspectos de la religiosidad e involucramiento religioso, identifica 17 dimensiones diferentes que pueden medirse, estas incluyen: creencia religiosa, afiliación religiosa, práctica religiosa (pública y privada), motivación religiosa, afrontamiento religioso, apoyo religioso, entre otras. La falta de acuerdo entre las diferentes disciplinas que estudian la religiosidad, e incluso al interior de ellas, así como el debate en torno a las dimensiones que debería considerarse hace más compleja la medición (Lemos, Gore, PugaGonzalez, \& Shults, 2019).

Una de las escalas más influyentes para medir involucramiento religioso ha sido la escala de orientación religiosa (ROS) de Allport y Ross (1967), pioneros en este campo de estudio (Zwingmann, Klein, \& Büssing, 2011). Koenig (2018) sitúa su trabajo dentro de la dimensión de motivación religiosa. Originalmente consiste en una versión de 21 ítems, divididos en dos dimensiones: orientación intrínseca y extrínseca. Sobre la base de ella, Gorsuch y Venable (1983) confeccionan una escala más breve, utilizando un lenguaje más simple, esperando que sea más aplicable a otros rangos de edad, variedad de países y en presencia o ausencia de afiliación religiosa. Su primera versión es la Age Universal I-E de 20 ítems. Posteriormente, surge una propuesta de 14 ítems de esta última escala, que subdivide la escala extrínseca en dos, orientación extrínseca social y orientación extrínseca personal (Gorsuch $\&$ McPherson, 1989). Finalmente, se propone la Age Universal I-E de 12 ítems, que conserva la propuesta de tres dimensiones (Maltby, 1999, 2002).

Las opciones de respuesta también han recibido atención, considerándose que la de tres opciones de respuesta es suficientemente útil (Sí, A veces, No) y más aplicable que la opción Likert a personas no religiosas (Maltby, 2002). No obstante, se continúa utilizando esta familia de escalas en sus diferentes versiones y tipos de respuesta. 
Un punto destacable es que la escala, en sus diferentes versiones, se haya usado en adolescentes sin haber sido exhaustivamente validada para esta población (Ens \& Bond, 2007; Greening \& Stoppelbein, 2002; Holder et al., 2000; Pössel et al., 2011). Gorsuch y Venable (1983) validaron su escala de 20 ítems, en dos submuestras de escuelas religiosas, ninguna de las cuales supera los 260 participantes. incluso si se reanalizaran los datos esto dificulta calcular los indicadores de bondad de ajuste adecuadamente. Una muestra sobre 500 e idealmente sobre 1000 participantes es lo recomendado para hacer esto (Kim, 2005). Maltby (1999), en su validación general de la versión de 12 ítems, incluye en su muestra de 3390 participantes a 567 escolares entre 12 y 18 años. No obstante, dos limitaciones importantes deben tenerse presentes: 1) esta muestra de adolescentes, así como la general, está compuesta de diferentes muestras parciales. 2) No analiza, ni en este ni en ningún otro artículo, la estructura especifica de las dimensiones en adolescentes. En un estudio previo sólo analiza la fiabilidad, pero no la estructura de la escala (Maltby, 1997). Si bien posteriormente se ha realizado al menos un estudio no publicado que analiza sus dimensiones en adolescentes, que encuentra solamente dos dimensiones con un buen ajuste (Banister, Possel, \& Adelson, 2011, como se citó en Banister, 2011).

Un elemento clave para considerar una posible diferencia según el ciclo vital de los participantes es la teoría del desarrollo de la Fe, que está a la base del instrumento (Allport,1950). En ella se considera tres fases: 1) Cruda credulidad, durante la preadolescencia donde se cree sin cuestionar lo que procede de los padres y figuras de autoridad. 2) Satisfacción del racionalismo: se genera durante la adolescencia en ella se comienza a cuestionar el sistema de creencias que han recibido. 3) Madurez religiosa: se genera en la adultez y en ella se revisarían el sistema de creencias religiosas, manteniendo aquello que brinda significado a la vida y descartando lo que no lo hace. Folwer (1981) posteriormente presenta una teoría más compleja del desarrollo de la fe en siete fases: 1) Fe primaria (infancia temprana hasta los 2 años). 2) $\mathrm{Fe}$ intuitiva-proyectiva (infancia temprana). 3) Fe mítica-literal (infancia media) 4) Fe sintético-convencional (adolescencia en adelante). 5) fe individual-reflexiva (adultez joven en adelante). 6) Fe conjuntiva (edad media en adelante). 7) Fe universalizada (mediana edad en adelante). Todas estas etapas se consideran dentro del contexto bio-sociocultural en que los sujetos se encuentran. De acuerdo a esta teoría, se asume que debería haber diferencias a lo largo del ciclo vital en el involucramiento religioso, avanzando secuencialmente hacia una fe más madura, si bien no hay garantía de progresión de las fases (Folwer \& Dell, 2004, 2006).

En Latinoamérica la escala Age Universal I-E 12 ha sido aplicada frecuentemente en universitarios, incluido Chile (Carrasco, 2012; Simkin \& Etchezahar, 2013; Rivera-Ledesma, Zavala-Jiménez, \& Montero-López, 2016). No obstante, esta escala no ha sido validada en población infanto-juvenil. La utilidad de contar con esta escala en un rango etario más amplio es clara si se considera que el involucramiento religioso puede considerarse un factor protector de salud mental en la población infanto-juvenil, entre otras ventajas (Holmes \& Kim-Spoon, 2015; Neymotin \& Downing-Matibag, 2013; Rew \& Wong, 2006; Sharma, Mustanski, Dick, Bolland, \& Kertes, 2019; Schreiber \& Culbertson, 2014). Estudiar la religiosidad en el contexto escolar tiene especial valor dado el proceso de socialización que puede promover o desincentivar el involucramiento religioso (Barrett, Pearson, Muller, \& Frank, 2007). La menor atención que ha recibido el involucramiento religioso en la población infanto-juvenil limita el avance en la compresión de los niños, niñas y adolescentes sobrevivientes de experiencias traumáticas, asî como quienes presentan victimización de pares, conductas sexuales de riesgo y consumo de sustancias (Bryant-Davis, Ellis, Burke-Maynard, Moon, Counts, \& Anderson, 2012; Helms et al., 2014).

Este estudio tiene como objetivo validar la Age Universal I-E 12, analizando la estructura factorial que presenta a través de una estrategia metodológica más compleja que la que se ha venido utilizando habitualmente y que no da cuenta de todos los aspectos que actualmente deben considerarse al revisar las propiedades psicométricas de un instrumento. La mayoría de los autores ha analizado la estructura factorial de esta escala mediante el análisis de componentes principales, excepto Cohen et al. (2017) quienes lo descartan por suponer que la medición de los ítems está libre de error. En la presente investigación se coincide con este criterio, si bien en vez de utilizar exclusivamente Análisis factorial confirmatorio (CFA) 
como lo hacen ellos, se opta por usar ecuaciones estructurales exploratorias (ESEM) y CFA. Se ha propuesto que ESEM es una estrategia más adecuada que AFE dada su flexibilidad para considerar las cargas factoriales más cercanas a como se dan en la realidad, al permitir que un mismo ítem cargue en más de un factor (Marsh, Nagengast, \& Morin, 2013). Una comparación entre ESEM y CFA puede resultar muy útil para comprender la estructura factorial de una escala al permitir una perspectiva más completa (Howard, Gagné, \& Forest, 2018; Marsh Morin, Parker, \& Kaur, 2014). Con todo, si ambas soluciones no se diferencian es preferible mantener la estructura reflejada por el CFA dada su mayor facilidad de interpretación Booth y Hughes (2014).

\section{METODOLOGÍA}

Es un tipo de estudio no experimental, puesto que no se manipulan variables. El diseño es transeccional correlacional (Hernández, Fernández, \& Baptista, 2010).

\section{MUESTRA}

Participaron inicialmente 3962 estudiantes de ambos sexos entre cuarto año de enseñanza básica y cuarto año de enseñanza media, provenientes de diferentes establecimientos de Arica. Se incluyen escuelas públicas, subvencionadas y privadas. Se descartaron 105 casos que tenían más de un $25 \%$ del instrumento sin responder. El rango de edad fue de 8 a 19 años.

\section{PROCEDIMIENTO}

1. Obtención de la aprobación del Comité de Ética de la Universidad de Tarapacá. Este estudio es parte de un proyecto mayor del Centro de Justicia Educacional.

2. Muestreo piloto con 223 estudiantes en la ciudad de Antofagasta para verificar la adecuada comprensión de los ítems. Se solicitó consentimiento a padres y asentimiento a los estudiantes.

3. Se contactó e invitó a 42 establecimientos educacionales de la ciudad de Arica a participar del estudio. Un 69\% accedió a participar del estudio, considerándose 29 establecimientos en total.

4. Se solicitó los consentimientos a los padres y posteriormente el asentimiento informado a los estudiantes.

5. Cada aplicación se realizó dentro de 20 minutos en forma grupal al interior de la sala curso.
Al menos dos encuestadores están presentes por sala para responder las dudas, aparte del profesor del mismo establecimiento.

\section{INSTRUMENTOS}

Age Universal I-E-12 (Maltby, 1999, 2002; Gorsuch \& Venable, 1983, 1989): Consta de 12 ítems, seis de los cuales evalúan la orientación religiosa (I), tres la Orientación extrínseca personal (EP) y tres la orientación extrínseca social (ES). Se opta por el formato de respuesta sugerido en la adaptación de Maltby (2002), tipo Likert con tres opciones de respuesta, que son 1, (sí), 2 (a veces) y 3 (No). El rango total de puntaje va de 12 a 36. Se utilizó la traducción de la validación chilena con universitarios, la que contó con una aplicación piloto para verificar su pertinencia (Carrasco, 2012).

\section{ANÁLISIS ESTADÍSTICOS}

Se utiliza el programa SPSS versión 21 para obtener los valores descriptivos y realizar comparaciones entre grupos y el programa MPLUS versión 7.4 para realizar análisis de ecuaciones estructurales exploratorias (ESEM) y análisis factorial confirmatorio (CFA) (Asparouhov \& Muthén, 2009; Muthén \& Muthén, 2017). Se opta por una rotación oblicua dado que es lo recomendado en ciencias sociales (Lloret-Segura, Ferreres-Traver, Hernández-Baeza, \& Tomás-Marco, 2014). La rotación seleccionada es geomin.

El análisis se realizó en cuatro fases:

I. Estadísticos descriptivos.

II. Comparación de grupos por sexo y grado.

III. Análisis factorial del instrumento con uno, dos y tres factores.

IV. Fiabilidad del instrumento.

Al ser tres las opciones de respuesta se consideró los datos como categóricos y se utilizó el estimador de Mínimos Cuadrados Ponderados Robustos o por sus siglas en inglés WLMSV (Brown, 2015; Li, 2016).

\section{RESULTADOS}

\section{Fase I: Estadísticos descriptivos Participantes}

La muestra final estuvo constituida por 3857 estudiantes entre cuarto básico y cuarto medio. Un $50,7 \%$ fueron mujeres y un $49,3 \%$ fueron hombres. La edad media fue de 12,7 años y la desviación estándar de 2,6. El 91,7\% de los 
estudiantes fue de nacionalidad chilena, un $4 \%$ fue de Bolivia, un 2,7\% de Perú y el resto se distribuyó en otras nacionalidades. Respecto a la religión, un $47,9 \%$ se auto clasifica como católico, $8,9 \%$

Tabla 1. Distribución de los participantes de la muestra según curso.

\begin{tabular}{ccc}
\hline & Frecuencia & Porcentaje \\
\hline BÁSICA & 553 & 14,3 \\
$4^{\circ}$ & 490 & 12,7 \\
$5^{\circ}$ & 474 & 12,3 \\
$6^{\circ}$ & 525 & 13,6 \\
$7^{\circ}$ & 449 & 11,6 \\
$8^{\circ}$ & &
\end{tabular}

MEDIA

$\begin{array}{cccc} & 1^{\circ} & 437 & 11,3 \\ & 2^{\circ} & 346 & 9,0 \\ & 3^{\circ} & 319 & 8,3 \\ & 4^{\circ} & 264 & 6,8 \\ \text { Total } & & 3857 & 100,0\end{array}$

evangélico, $11,3 \%$ de otras religiones y un $32 \%$ de ninguna. En cuanto a la etnia, un $25,6 \%$ fue Aymara, La distribución de los cursos se muestra en la tabla $1 \mathrm{y}$ puede apreciarse que es bastante homogénea.

Los estadísticos descriptivos de cada ítem se muestran en la tabla 2. Puede apreciarse que la asimetría y curtosis se mantienen en rangos aceptables para considerar que se distribuyen normalmente (Ryu, 2011).

\section{Fase II: Comparación de grupos por sexo y grado}

Se realizó un ANOVA factorial para comparar grupos por sexo (mujer/hombre) y grado (primaria/ secundaria), sexo, considerando el puntaje total de involucramiento religioso como variable dependiente.

Las estudiantes mujeres obtuvieron una media de $20,5(D E=6,6)$ y los hombres obtuvieron 20 $(D E=6,7)$. Los estudiantes de primaria tuvieron una media de involucramiento religioso de 23,3 ( $D E$ $=6,3$ ) mientras que los de secundaria obtuvieron $18,7(D E=6,1)$. El estadístico de Levene $(3,4, p$ $=0,78)$ permite concluir que las varianzas de los grupos pueden considerarse iguales. Se encontró un efecto principal significativo sólo para el grado de los estudiantes, $F(3,3853)=616,15, p<0,000$. También se encontró un efecto de interacción de sexo y grado, $F(3,3853)=176,27, p<0,031$. Los

Tabla 2. Estadísticos descriptivos de cada ítem.

\begin{tabular}{|c|c|c|c|c|c|c|}
\hline & Mínimo & Máximo & $M$ & $D S$ & Asimetría & Curtosis \\
\hline Iteml & 1,0 & 3,0 & 1,67 &, 730 &, 610 &,- 916 \\
\hline Item2 & 1,0 & 3,0 & 1,41 & ,685 & 1,376 & ,468 \\
\hline Item3 & 1,0 & 3,0 & 1,75 &, 786 & ,467 & $-1,238$ \\
\hline Item4 & 1,0 & 3,0 & 1,85 & 822 & ,289 & $-1,463$ \\
\hline Item5 & 1,0 & 3,0 & 1,95 & ,832 &, 088 & $-1,549$ \\
\hline Item 6 & 1,0 & 3,0 & 1,73 &, 809 &, 536 & $-1,272$ \\
\hline Item 7 & 1,0 & 3,0 & 1,82 &, 837 & ,343 & $-1,489$ \\
\hline Item8 & 1,0 & 3,0 & 1,85 & 847 &, 300 & $-1,541$ \\
\hline Item9 & 1,0 & 3,0 & 1,89 &, 838 & ,210 & $-1,546$ \\
\hline Item 10 & 1,0 & 3,0 & 1,33 & ,632 & 1,719 & 1,602 \\
\hline Item 11 & 1,0 & 3,0 & 1,54 & ,740 & ,966 &,- 528 \\
\hline Item12 & 1,0 & 3,0 & 1,45 &, 721 & 1,247 & 037 \\
\hline
\end{tabular}


hombres obtienen los puntajes de media extremos de involucramiento religioso, siendo su media la más alto de primaria con $23,4(D E=6,4)$ y la más baja de secundaria con 17,9 $(D E=6)$.

\section{Fase III: Análisis factorial del instrumento}

Primero, se replica en análisis de componentes principales utilizado por todos los autores que han analizado la escala. No obstante, solamente se hace con el fin de comparar este análisis con el obtenido posteriormente mediante ESEM y CFA, puesto que el análisis de componentes principales no es realmente un análisis factorial. Los resultados se muestran en la tabla 3. Si se considera el resultado del análisis de componentes principales puede observarse que sugiere dos dimensiones. En la primera de ellas se combina la dimensión intrínseca con la extrínseca personal. No obstante, para decidir si este modelo es aceptable es preciso considerar la bondad de ajuste. Esto puede apreciarse de mejor manera mediante el análisis realizado a continuación mediante Mplus.
La correlación entre ambos factores es 0,53 lo que sugiere factores relacionados pero independientes. Si este análisis se realiza con extracción de mínimos cuadrados no ponderados, el cual si es propiamente análisis factorial, y con rotación oblimin, aunque los factores obtienen la misma distribución, la correlación entre ellos es de 0,62.

En la tabla 4 comparan los resultados obtenidos a partir del ESEM y CFA, desde 1 a 3 factores. Es importante tener en cuenta que el ESEM sugiere los ítems libremente, en tanto que primero el CFA se ocupa para 2 factores diferenciando clásicamente entre intrínseco y extrínseco. Luego se plantea un CFA alternativo, dejando combinados intrínseco y extrínseco personal. Finalmente, en el CFA de 3 factores, el factor extrínseco se separa en social y personal.

Tabla 3. Análisis de componentes principales de la escala I-E 12.

\begin{tabular}{|c|c|c|c|}
\hline & Ítem & 1 & 2 \\
\hline \multicolumn{4}{|c|}{ Orientación Intrínseca } \\
\hline 1 & Disfruto leyendo o instruyéndome acerca de mi creencia religiosa & 0,641 & 0,167 \\
\hline 3 & Es importante para mí pasar tiempo en meditación privada y/u oración & 0,652 & 0,025 \\
\hline 4 & He tenido frecuentemente una fuerte sensación de la presencia de Dios & 0,744 & $-0,005$ \\
\hline 6 & Me esfuerzo por vivir toda mi vida de acuerdo a mis creencias religiosas & 0,712 & 0,132 \\
\hline 8 & Mi religión es importante porque me da respuestas a muchas preguntas sobre el sentido de la vida & 0,786 & 0,050 \\
\hline 11 & Mi forma de ver la vida está basada en mi religión & 0,562 & 0,280 \\
\hline \multicolumn{4}{|c|}{ Orientación extrínseca social } \\
\hline 2 & Voy a la iglesia o culto religioso porque me ayuda a hacer amigos & 0,087 & 0,773 \\
\hline 10 & Voy a la iglesia o culto religioso principalmente para pasar tiempo con mis amigos. & $-0,071$ & $\mathbf{0 , 8 9 9}$ \\
\hline 12 & Voy a la iglesia o culto religioso, sobre todo porque me gusta ver a la gente que conozco allí. & 0,083 & $\mathbf{0 , 8 0 4}$ \\
\hline \multicolumn{4}{|c|}{ Orientación extrínseca personal } \\
\hline 5 & Oro o rezo principalmente para tener alivio y protección & 0,876 & $-0,162$ \\
\hline 7 & Lo que más me ofrece la religión es consuelo en tiempos de problemas y angustias & 0,773 & $-0,019$ \\
\hline 9 & Oro o medito para obtener paz y felicidad. & 0,827 & $-0,070$ \\
\hline
\end{tabular}

Nota 1. Se realizó rotación Oblimin.

Nota 2. Se han resaltado las cargas factoriales mayores a 0,3 según el factor que las agrupa. 
Tabla 4. Modelos ESEM y CFA de la muestra total.

\begin{tabular}{|c|c|c|c|c|c|c|c|c|c|c|c|c|c|}
\hline \multirow[t]{3}{*}{ Ítem } & \multirow{3}{*}{$\begin{array}{l}1 \text { factor } \\
\text { CFA 1D }\end{array}$} & \multicolumn{6}{|c|}{2 factores } & \multicolumn{6}{|c|}{3 factores } \\
\hline & & \multicolumn{2}{|c|}{ ESEM } & \multicolumn{2}{|c|}{ CFA clásico } & \multicolumn{2}{|c|}{ CFA alter } & \multicolumn{3}{|c|}{ ESEM } & \multicolumn{3}{|c|}{ CFA } \\
\hline & & F1 & F2 & INT & EXT & INC & EXS & F1 & F2 & F3 & INT & EXP & EXS \\
\hline 1 & 0.783 & 0.669 & 0.166 & 0.793 & & 0.793 & & 0.726 & 0.050 & 0.035 & 0.795 & & \\
\hline 2 & 0.766 & 0.181 & 0.707 & & 0.774 & & 0.870 & 0.023 & 0.094 & 0.765 & & & 0.870 \\
\hline 3 & 0.680 & 0.665 & 0.036 & 0.688 & & 0.690 & & 0.373 & 0.315 & 0.041 & 0.692 & & \\
\hline 4 & 0.759 & 0.775 & -0.003 & & 0.770 & 0.770 & & 0.323 & 0.453 & 0.042 & 0.772 & & \\
\hline 5 & 0.806 & 0.999 & -0.239 & 0.815 & & 0.814 & & 0.002 & 0.940 & -0.055 & & 0.840 & \\
\hline 6 & 0.845 & 0.740 & 0.154 & & 0.858 & 0.855 & & 0.927 & -0.028 & -0.047 & 0.857 & & \\
\hline 7 & 0.790 & 0.801 & 0.004 & 0.801 & & 0.800 & & 0.630 & 0.261 & -0.078 & & 0.831 & \\
\hline 8 & 0.863 & 0.812 & 0.085 & & 0.877 & 0.872 & & 0.882 & 0.082 & -0.093 & 0.875 & & \\
\hline 9 & 0.818 & 0.925 & -0.128 & 0.827 & & 0.827 & & 0.203 & 0.665 & 0.014 & & 0.856 & \\
\hline 10 & 0.766 & -0.006 & 0.901 & & 0.773 & & 0.849 & -0.116 & 0.008 & 0.987 & & & 0.850 \\
\hline 11 & 0.818 & 0.643 & 0.245 & 0.831 & & 0.830 & & 1.007 & -0.202 & 0.020 & 0.831 & & \\
\hline 12 & 0.796 & 0.140 & 0.786 & & 0.804 & & 0.906 & 0.103 & -0.011 & 0.812 & & & 0.906 \\
\hline
\end{tabular}

Nota 1. Se han resaltado las cargas factoriales mayores a 0,3 en negrita para facilitar reconocer el factor que las agrupa.

Nota 2. CFA clásico: es el propuesto teóricamente, CFA Alter: combina intrínseca con extrínseca personal. INT: intrínseca clásica, EXT: extrínseca clásica, INC: intrínseca combinada con extrínseca personal, EXS: extrínseca social. EXP: extrínseca personal.

En la tabla 5 se presentan las correlaciones entre los factores. Puede apreciarse que, al considerar el CFA para dos factores, dividiendo en intrínseco y extrínseco la covarianza es demasiado elevada como para considerar que son realmente dos factores.
En la tabla 6 se presentan también los análisis ESEM y CFA para las submuestras de primaria, de cuarto a sexto grado, y secundaria, desde séptimo básico en adelante, para descartar que exista una estructura factorial diferente en estos dos grupos. Estas contaron con 1517 y 2340

Tabla 5. Resumen de correlaciones entre los factores.

\begin{tabular}{|c|c|c|c|c|c|c|c|c|c|c|c|}
\hline \multicolumn{2}{|c|}{ ESEM 2F } & \multicolumn{2}{|c|}{ CFA Clas 2F } & \multicolumn{2}{|c|}{ CFA 2 F alter } & \multicolumn{3}{|c|}{ ESEM 3F } & \multicolumn{3}{|c|}{ CFA 3F } \\
\hline \multirow{3}{*}{ F1 } & F2 & & EXT & & EXS & & $\mathrm{F} 2$ & F3 & & EXP & EXS \\
\hline & 0.669 & INT & 0.951 & INC & 0,756 & F1 & 0.841 & 0.771 & INT & 0.944 & 0.779 \\
\hline & & & & & & F2 & & 0.598 & EXP & & 0.675 \\
\hline
\end{tabular}

Nota 1. CFA Clas: distribución bidimensional clásica según la teoría, CFA Alter: combina intrínseca con extrínseca personal. INT: intrínseca clásica, EXT: extrínseca clásica, INC: intrínseca combinada con extrínseca personal, EXS: extrínseca social. EXP: extrínseca personal. 
casos respectivamente. Asimismo, se presentan las submuestras separadas por afiliación religiosa, incluyendo 2622 con alguna afiliación y 1235 sin afiliación religiosa. Puede apreciarse en la tabla 6 que la distribución es la misma en ambas clasificaciones (Grado y afiliación) que en el ESEM de 2 factores sobre la muestra total. Esto permite descartar que la estructura factorial varíe.

El análisis de los indicadores de bondad de ajuste se presenta en la tabla 7. Por convención un error de Aproximación Cuadrático Medio (RMSEA) bajo .08 es aceptable y bajo .06 es

Tabla 6. ESEM y CFA por submuestras.

\begin{tabular}{|c|c|c|c|c|c|c|c|c|c|c|c|c|c|c|c|c|}
\hline \multirow{4}{*}{ Ítem } & \multicolumn{8}{|c|}{ Grupo curso } & \multicolumn{8}{|c|}{ Afiliación religiosa } \\
\hline & \multicolumn{4}{|c|}{ ESEM } & \multicolumn{4}{|c|}{ CFA Alter } & \multicolumn{4}{|c|}{ ESEM } & \multicolumn{4}{|c|}{ CFA Alter } \\
\hline & \multicolumn{2}{|c|}{ Primaria } & \multicolumn{2}{|c|}{ Secundaria } & \multicolumn{2}{|c|}{ Primaria } & \multicolumn{2}{|c|}{ Secundaria } & \multicolumn{2}{|c|}{ Religioso } & \multicolumn{2}{|c|}{ No religioso } & \multicolumn{2}{|c|}{ Religioso } & \multicolumn{2}{|c|}{ No religioso } \\
\hline & F1 & $\mathrm{F} 2$ & F1 & F2 & INC & EXS & INC & EXS & F1 & F2 & F1 & F2 & INC & EXS & INC & EXS \\
\hline 1 & 0.610 & 0.176 & 0.686 & 0.145 & 0.728 & & 0.797 & & 0.658 & 0.120 & 0.556 & 0.335 & 0.743 & & 0.837 & \\
\hline 2 & 0.193 & 0.663 & 0.195 & 0.727 & & 0.824 & & 0.904 & 0.180 & 0.693 & 0.055 & 0.832 & & 0.854 & & 0.876 \\
\hline 3 & 0.591 & 0.076 & 0.704 & -0.006 & 0.639 & & 0.698 & & 0.641 & 0.030 & 0.655 & 0.064 & 0.661 & & 0.701 & \\
\hline 4 & 0.683 & -0.016 & 0.781 & 0.004 & 0.665 & & 0.782 & & 0.725 & 0.007 & 0.850 & -0.054 & 0.729 & & 0.796 & \\
\hline 5 & 0.901 & -0.247 & 1.007 & -0.225 & 0.718 & & 0.836 & & 0.931 & -0.238 & 1.126 & -0.313 & 0.759 & & 0.846 & \\
\hline 6 & 0.704 & 0.154 & 0.754 & 0.123 & 0.805 & & 0.847 & & 0.734 & 0.116 & 0.677 & 0.275 & 0.816 & & 0.903 & \\
\hline 7 & 0.703 & 0.006 & 0.840 & 0.010 & 0.700 & & 0.846 & & 0.749 & -0.016 & 0.779 & 0.094 & 0.735 & & 0.849 & \\
\hline 8 & 0.754 & 0.060 & 0.828 & 0.083 & 0.788 & & 0.890 & & 0.801 & 0.059 & 0.748 & 0.172 & 0.841 & & 0.883 & \\
\hline 9 & 0.831 & -0.104 & 0.954 & -0.167 & 0.752 & & 0.828 & & 0.907 & -0.171 & 0.872 & 0.005 & 0.784 & & 0.868 & \\
\hline 10 & -0.007 & 0.871 & -0.007 & 0.923 & & 0.791 & & 0.875 & -0.008 & 0.882 & -0.009 & 0.950 & & 0.824 & & 0.924 \\
\hline 11 & 0.620 & 0.266 & 0.656 & 0.204 & 0.804 & & 0.812 & & 0.650 & 0.200 & 0.536 & 0.418 & 0.795 & & 0.893 & \\
\hline 12 & 0.177 & 0.730 & 0.127 & 0.813 & & 0.877 & & 0.917 & 0.128 & 0.783 & 0.124 & 0.815 & & 0.887 & & 0.939 \\
\hline
\end{tabular}

Nota 1. Se han resaltado las cargas factoriales mayores a 0,3 en negrita para facilitar reconocer el factor que las agrupa.

Nota 2. CFA Alter: combina intrínseca con extrínseca personal. INT: intrínseca clásica, EXT: extrínseca clásica, INC: intrínseca combinada con extrínseca personal, EXS: extrínseca social. EXP: extrínseca personal.

bueno, mientras que un Ajuste Comparativo (CFI) y el Índice Tucker-Lewis (TLI) mayor a .9 es adecuado y sobre 95 es óptimo (Brown, 2015; Hu \& Bentler, 1999; McDonald \& Ho, 2002). El chi cuadrado no se considera actualmente un indicador imprescindible dado los problemas que presenta con muestras mayores a 200 y en casos de distribución no normal. El ratio $\chi 2$ / df se considera adecuado bajo 3, si bien está sujeto a la misma limitación que el Chi cuadrado en muestras grandes (Brown, 2015; Hu \& Bentler, 1999).

Puede notarse que en todos los modelos el CFI y TLI son óptimos, siendo el RMSEA el índice de bondad de ajuste que es más difícil de satisfacer. La solución del CFA 2 factores alternativa si lo satisface. En todo caso, los índices de modificación sugieren covarianza de errores entre los ítems 5,9, 10 y 12 , lo que implica que eliminar alguno de ellos podría mejorar el ajuste del modelo.

\section{Invarianza por sexo}

Se analizó mediante CFA multigrupo la invarianza por sexo (Mujer/Hombre). Los resultados pueden observarse en la tabla 8 , siguiendo las recomendaciones de Putnick y Bornstein (2016) para reportar invarianza. Estos son niveles más frecuentes y útiles de informar (Xing \& Hall, 2015). Dado que los datos son categóricos, en vez de $\Delta \chi 2$ se utilizó $\Delta \chi 2 \mathrm{mv}$, basado en la escala corregida Satorra-Bentler (Sarorra, 2000), que en MPLUS se denomina DIFFTEST. Se encuentra 
Tabla 7. Indicadores de bondad de ajuste de los diferentes modelos.

\begin{tabular}{|c|c|c|c|c|c|c|c|c|}
\hline & $\chi^{2}$ & DF & $\chi^{2} / \mathrm{df}$ & $\chi^{2}$ P-Value & CFI & TLI & RMSEA & RMSEA 90\% IC \\
\hline ESEM 1 Factor & 2750.608 & 54 & 50,94 & 0,000 & 0.957 & 0.947 & 0.114 & $0.110-0.117$ \\
\hline ESEM 2 Factores & 582.675 & 43 & 13,55 & 0,000 & 0.991 & 0.987 & 0.057 & $0.053-0.061$ \\
\hline CFA 2 Factores clásico & 2660.176 & 53 & 50,19 & 0,000 & 0.958 & 0.948 & 0.113 & $0.109-0.117$ \\
\hline ESEM 3 Factores & 188,043 & 33 & 5,69 & 0,000 & 0,998 & 0,995 & 0,035 & $0,030-0,040$ \\
\hline CFA 3 Factores clásico & 2545.776 & 51 & 49,92 & 0,000 & 0.960 & 0.948 & 0,113 & $0.109-0.116$ \\
\hline CFA 2 Factores alternativo & 846.278 & 53 & 15,96 & 0,000 & 0.987 & 0.984 & 0.062 & $0.059-0.066$ \\
\hline ESEM 2 Factores Primaria & 190.741 & 43 & 4,44 & 0,000 & 0.989 & 0.984 & 0.048 & $0.041-0.055$ \\
\hline CFA 2 Factores alter Primaria & 361.274 & 53 & 6,82 & 0,000 & 0.978 & 0.973 & 0.062 & $0.056-0.068$ \\
\hline ESEM 2 Factores Secundaria & 486.520 & 43 & 11,31 & 0,000 & 0.988 & 0.981 & 0.066 & $0.061-0.072$ \\
\hline CFA 2 Factores alter Secundaria & 574.653 & 53 & 10,84 & 0,000 & 0.986 & 0.982 & 0.065 & $0.060-0.070$ \\
\hline ESEM 2 Factores religiosos & 460.497 & 43 & 10,71 & 0,000 & 0.987 & 0.979 & 0.061 & $0.056-0.066$ \\
\hline CFA 2 Factores alter religiosos & 312.043 & 53 & 5,89 & 0,000 & 0.989 & 0.987 & 0.063 & $0.056-0.070$ \\
\hline ESEM 2 Factores NO religiosos & 182.102 & 43 & 4,23 & 0,000 & 0.994 & 0.991 & 0.051 & $0.044-0.059$ \\
\hline $\begin{array}{l}\text { CFA } 2 \text { Factores alter NO } \\
\text { religiosos }\end{array}$ & 624.338 & 53 & 11,77 & 0,000 & 0.982 & 0.977 & 0.064 & $0.060-0.069$ \\
\hline
\end{tabular}

Nota 1. $\chi 2$ : Chi cuadrado. DF: grados de libertad. $\chi^{2} / \mathrm{df}$ : ratio chi cuadrado. Nota 2: tanto en ESEM 1 factor inicial como final los resultados son lógicamente los mismos que en CFA al ser unidimensionales.

Nota 2. Alter: alternativo.

evidencia favorable a la invarianza tanto a nivel de configuración, métrica como escalar. Los puntos de corte de la bondad de ajuste coinciden con los propuestos por Chen (2007), quien considera que los criterios claves para determinar invarianza no incluyen los basado en $\Delta \chi 2$, sino que propone que CFI puede variar hasta .010, RMSEA hasta .015. De todas maneras, se cumple con el criterio de DIFFEST ( $\mathrm{p} \geq .05$ ), que indica que no existen diferencias significativas entre los grupos y por ende existe invarianza.

Tabla 8. Indicadores de invarianza por sexo.

\begin{tabular}{|c|c|c|c|c|c|c|c|c|c|c|c|}
\hline Modelo & $\chi^{2}(d f)$ & CFI & $\begin{array}{c}\text { RMSEA (90\% } \\
\text { IC) }\end{array}$ & WRMR & $\begin{array}{l}\text { Modelo } \\
\text { referencia }\end{array}$ & $\Delta \chi^{2} M V$ & $\begin{array}{c}\mathbf{P} \\
\text { DIFFTEST }\end{array}$ & $\Delta \mathrm{CFI}$ & ARMSEA & DWRMR & Decisión \\
\hline $\begin{array}{l}\text { M1: } \\
\text { Invarianza } \\
\text { configuración }\end{array}$ & $\begin{array}{c}752.300 \\
(118)\end{array}$ & 0.990 & $\begin{array}{c}0.053(0.049- \\
0.056)\end{array}$ & 2.492 & -- & & -- & -- & -- & -- & -- \\
\hline $\begin{array}{l}\text { M2: } \\
\text { Invarianza } \\
\text { métrica }\end{array}$ & $\begin{array}{l}668.710 \\
(128)\end{array}$ & 0.991 & $\begin{array}{c}0.047(0.043- \\
0.050)\end{array}$ & 2.567 & M1 & 18.250 & .0509 & 001 & ,006 & ,075 & Acepta \\
\hline $\begin{array}{l}\text { M3: } \\
\text { Invarianza } \\
\text { escalar }\end{array}$ & $\begin{array}{c}551.050 \\
(140)\end{array}$ & 0.993 & $\begin{array}{c}0.039(0.036- \\
0.042)\end{array}$ & 2.600 & M2 & 11.328 & .5010 &, 002 & ,008 &, 033 & Acepta \\
\hline
\end{tabular}




\section{Fase IV: Fiabilidad del instrumento y sus escalas}

La fiabilidad de la escala total fue de .91. La fiabilidad de la dimensión combinada intrínseca fue de .91 y de la dimensión extrínseca social fue de ,81. Según Nunally (1987) y Morales (2007) la fiabilidad de las escalas se considera adecuada a partir de .70 .

\section{DISCUSIÓN}

El presente trabajo tuvo como propósito validar la Age Universal I-E 12, analizando la estructura factorial que presenta a través de una estrategia metodológica más compleja que la que se ha venido utilizando habitualmente y que no da cuenta de todos los aspectos que actualmente deben considerarse al revisar las propiedades psicométricas de un instrumento.

Utilizar el análisis de componentes principales para revisar la estructura factorial ha sido un aspecto cuestionable desde el inicio de la escala y en las validaciones del instrumento en Latinoamérica con universitarios (Carrasco, 2012; Maltby, 1999, 2002; Simkin \& Etchezahar, 2013; Rivera-Ledesma, Zavala-Jiménez, \& Montero-López, 2016). El hecho de que esta forma de análisis no considere el error le quita precisión. Al margen de que no se considera que realmente sea parte de la familia de análisis factorial (Hefetz \& Liberman, 2017; Lloret-Segura et al., 2014). Otro aspecto que no se ha tenido en cuenta regularmente es informar el RMSEA entre los indicadores de bondad de ajuste. Guiarse exclusivamente por CFI y TLI, cuando se reporta, resulta insuficiente tal y como se ha demostrado en los modelos revisados, puesto que no fue difícil cumplir con niveles óptimos de estos indicadores en ninguno de los mismos. La excepción es la validación argentina del instrumento donde si se reporta el RMSEA (Simkin \& Etchezahar, 2013). En tanto en México adicionalmente se opta por la rotación varimax en vez de utilizar una rotación oblicua como se recomienda en ciencias sociales (Lloret-Segura et al., 2014) y de hecho desde la propuesta original de Maltby (1999).

Sin duda, un aspecto central del presente trabajo es que los resultados cuestionan la tridimensionalidad del instrumento cuando es aplicado a escolares entre 8 y 19 años. La validación en universitarios en Chile fue realizada solo con 133 participantes, y aunque obtuvo tres factores no puede descartarse del todo que en una muestra mayor tenga variaciones o que al realizar un análisis como en el presente estudio eso varíe. No obstante, es factible suponer que el rango de edad sea lo decisivo para que surja esta diferencia en su dimensionalidad.

La estructura bifactorial del modelo que combina la dimensión intrínseca con la extrínseca personal tiene bastante lógica. De hecho, es discutible que los ítems de esta última dimensión midan algo extrínseco, puesto que más bien abordan el adscribirse a la fe por obtener algún tipo de recompensa (alivio, consuelo, felicidad) que realmente no es externa sino interna. Concordamos con Cohen et al. (2017) quienes la denominan como "utilitaria", puesto que efectivamente parece ser ese su foco. Ahora bien, esta dimensión puede diferenciarse en términos de bondad de ajuste en el modelo de tres factores, cumpliendo niveles óptimos en todos los indicadores. No obstante, la correlación entre los factores intrínseco y extrínseco personal es demasiado alta. En este sentido, al probar el CFA con dos y tres factores de acuerdo a la teoría, las correlaciones entre factores son sobre 0,95 , lo que hace totalmente innecesario suponer que sean factores diferenciables. De hecho, correlaciones entre factores sobre 0,80 indicarían que tiene más sentido considerar ambos factores en uno solo por el principio de parsimonia (Brown, 2015; Gunzler \& Morris, 2015). En cambio, al realizar el CFA con el modelo de dos dimensiones alternativo se obtiene indicadores de bondad de ajuste adecuados $($ RMSA $90 \% \mathrm{CI}=0.059>0.062>0.066, \mathrm{CFI}=$ 0.987 , TLI $=0.984)$. En este caso, la correlación entre ambos factores es 0,756 . Situándose debajo del límite. Ahora bien, aunque es aceptado que el WLSMV permite índices de ajuste más adecuados para datos categóricos, las correlaciones entre factores tienden a sobreestimarse ligeramente $(\mathrm{Li}$, 2016). Es necesario tener esto en cuenta, especialmente considerando que la correlación entre ambos factores al utilizar mínimos cuadrados no ponderados con rotación oblimin en SPSS arrojó 0,62 .

Esto sugiere que es necesario conocer si esta estructura bifactorial alternativa puede obtenerse en otros países latinoamericanos en este rango etario. Se ha encontrado diferencias en la distribución de la dimensionalidad en la versión de 14 ítems de Gorsuch y McPherson (1989) al compararla en universitarios bosnios musulmanes, serbios ortodoxos, eslovenos católicos 
y estadounidenses protestantes. Los únicos que obtuvieron tres dimensiones fueron estos últimos. En todos los demás se encontró que las dimensiones se combinaban como en la presente investigación, si bien utilizaron otra versión del instrumento, escala Likert de respuesta, una rotación varimax y un análisis de componentes principales (Flere, \& Lavrič, 2008). Adicionalmente, la correlación entre ambas dimensiones fue más alta en la muestra católica $(r=.66)$ que en las otras dos no protestantes. Si se considera que aproximadamente la mitad de nuestra muestra es católica, tiene sentido que la correlación sea la más similar a la del presente estudio. No obstante, esto contrasta parcialmente con los resultados obtenidos con una muestra polaca ( $95 \%$ de católicos) que utilizó el instrumento original (ROS) en que una solución de tres factores fue más adecuada (Brewczynski \& MacDonald, 2006).

La covarianza de los errores de algunos ítems (ej: 5, 9, 10 y 12) en el presente estudio sugiere que al ser eliminados algunos de ellos el ajuste podría mejorar. No obstante, se optó por mantener la totalidad de la escala dado que sus propiedades psicométricas parecen adecuadas.

El muestreo piloto de la presente investigación, permitió descartar dificultades en la comprensión de las preguntas. Esto es coherente con las modificaciones sucesivas que se propusieron de la escala de Allport y Ross (1967), que precisamente buscaban simplificar su leguaje para hacerlo aplicable a un rango de edad más amplio (Maltby, 1999, 2002).

Otro punto relevante es que tampoco se encuentre una estructura factorial diferente entre estudiantes de primaria y secundaria, lo que sería esperable especialmente si hubiese diferencias en la comprensión de los ítems. Si bien también sería esperable que la madurez de los estudiantes de secundaria pudiese afectar la estructura factorial. En todo caso, el hecho de que los estudiantes de primaria obtengan puntajes significativamente superiores a los de secundaria indica que en ese aspecto si existe diferencias entre ambos grupos. Esto puede sugerir que las intervenciones orientadas a promover el involucramiento religioso deberían empezar al menos desde primaria. El hecho de que la muestra presente no incluya estudiantes de los cursos iniciales, se debe a una limitante en la comprensión lectora de los estudiantes, que podría subsanarse en parte mediante un entrevistador que aplique individual en vez de colectivamente. No obstante, esto sin duda requiere más recursos para poder implementarse.

La presunción de que el involucramiento religioso se torna más maduro con la edad, presente tanto en la teoría de Allport (1950) como en Fowler (1981, Fowler \& Dell, 2004, 2006) puede presentar un desafío importante para la medición, especialmente dado que preguntas que abarquen conceptos más complejos necesariamente pueden requerir un proceso de pensamiento más sofisticado. Por ende, preguntas que sean también aplicables a niños y adolescentes pueden no tener la profundidad suficiente para medir esta madurez y preguntas que si lo hagan pueden no ser fácilmente comprensibles en menores de edad. Fowler ha sido criticado por presentar una teórica muy cognitiva (King \& Roeser, 2009). También es plausible que la madurez de la Fe pueda estar asociada más a un sentimiento personal que si pueda en alguna forma ser comparable a lo largo del ciclo vital.

La crítica sobre la aplicabilidad de la escala a sujetos no religiosos, que Maltby $(1999,2002)$ pretende superar al modificar las opciones de respuesta, no parece sustentarse en el presente estudio, dado que tanto religiosos como no religiosos obtienen la misma estructura factorial.

Un elemento constante de crítica a la escala Age Universal es que esta escala, y otras que midan involucramiento religioso, se dejen fuera otros aspectos de la espiritualidad. Sin duda, la diferenciación entre religión y espiritualidad ha cobrado bastante relevancia en los últimos años (Baumsteiger \& Chenneville, 2015). Esto sugiere que sería deseable complementar esta escala con otra que mida espiritualidad. Desde luego, la opción sería aplicar una escala que midiese ambas. No obstante, el hecho de que esta escala haya sido ampliamente estudiada al menos sugiere que puede tener sentido conservarla en la medición del involucramiento religioso.

La falta de diferencia por sexo en el puntaje total coincide con lo encontrado por Simkin y Etchezahar, (2013) en población de universitarios. Es posible que esto no varíe a lo largo de la edad de los sujetos, si bien sería sugerible contar con nuevas investigaciones en población infanto-juvenil para corroborarlo.

Una fortaleza de este estudio es el tamaño de la muestra, que incluso en todas las submuestras supera los 1000 sujetos. Considerando colegios 
tanto de filiación religiosa como sin filiación, lo que permite contar con mayor muestra de estudiantes no religiosos. No obstante, una limitación es que no se utilizó selección aleatoria, sino por conveniencia.

\section{CONCLUSIÓN}

La escala Age Universal I-E 12 en esta población infanto-juvenil mostró una estructural bifactorial, a diferencia de la trifactorial encontrada frecuentemente en edades mayores, combinándose la dimensión intrínseca con la extrínseca personal. Los indicadores de bondad de ajuste de la escala fueron adecuados. La fiabilidad de la escala también fue adecuada, tanto en la escala total como en las dos dimensiones obtenidas.

\author{
Agradecimientos \\ Esta investigación ha sido financiada por CONICYT PIA CIE160007.
}




\section{Referencias}

Allport, G. W. (1950). The Individual and His Religion: A Psychological Interpretation. New York, NY, US: Macmillan.

Allport, G. W. \& Ross, J. M. (1967). Personal religious orientation and prejudice. Journal of Personality and Social Psychology, $5,432-443$.

Asparouhov, T. \& Muthén, B. (2009). Exploratory structural equation modeling. Structural Equation Modeling, 16, 397-438. doi:1.1080/10705510903008204

Banister, A. W. (2011). Towards an improved measure of intrinsic/extrinsic religious motivation. Electronic Theses and Dissertations. Paper 67. https://doi.org/10.18297/etd/67

Barrett, J. B., Pearson, J., Muller, C., \& Frank, K. A. (2007), Adolescent Religiosity and School Contexts. Social Science Quarterly, 88, 1024-1037. doi:10.1111/j.1540-6237.2007.00516.x

Baumsteiger, R. \& Chenneville, T. (2015). Challenges to the Conceptualization and Measurement of Religiosity and Spirituality in Mental Health Research. Journal of Religion and Health, 54, 2344-2354.

Booth, T. \& Hughes D. J. (2014). Exploratory structural equation modeling of personality data. Assessment. 21(3), 260-71. doi: $10.1177 / 1073191114528029$

Brewczynski, J. \& MacDonald, D. A. (2006). Confirmatory Factor Analysis of the Allport and Ross Religious Orientation Scale With a Polish Sample. International Journal for the Psychology of Religion, 16(1), 63-76. doi:10.1207/s15327582ijpr1601_6

Brown, T. A. (2015). Methodology in the social sciences. Confirmatory factor analysis for applied research (2nd ed.). New York, NY, US: Guilford Press.

Bryant-Davis, T., Ellis, M. U., Burke-Maynard, E., Moon, N., Counts, P. A., \& Anderson, G. (2012). Religiosity, spirituality, and trauma recovery in the lives of children and adolescents. Professional Psychology: Research and Practice, 43(4), 306-314. http://dx.doi.org/10.1037/a0029282

Burke, A., Olphen, J.V., Eliason, M., Howell, R.G., \& Gonzalez, A. (2014). Re-examining Religiosity as a Protective Factor: Comparing Alcohol Use by Self-Identified Religious, Spiritual, and Secular College Students. Journal of Religion and Health, $53,305-316$.

Carrasco, C. A. (2012). Orientación religiosa y sintomatología depresiva en estudiantes de la Universidad del Bío-Bío. (Tesis de pregrado). Recuperado de http://repobib.ubiobio.cl/jspui/ handle/123456789/1546

Chen, F. F. (2007). Sensitivity of Goodness of Fit Indexes to Lack of Measurement Invariance. Structural Equation Modeling: A Multidisciplinary Journal, 14(3), 464-504. doi:10.1080/10705510701301834

Chen, Y. \& VanderWeele, T. J. (2018). Associations of Religious Upbringing With Subsequent Health and Well-Being From Adolescence to Young Adulthood: An Outcome-Wide Analysis. American journal of epidemiology, 187(11), 2355-2364. doi:10.1093/aje/kwy142

Chiswick, B. \& Mirtcheva, D. (2013). Religion and Child Health: Religious Affiliation, Importance, and Attendance and Health Status among American Youth. Journal of Family and Economic Issues, 34(1), 120-140. doi: 10.1007/s10834-012-9312-5

Cohen, A. B., Mazza, G. L., Johnson, K. A., Enders, C. K., Warner, C. M., Pasek, M. H., \& Cook, J. E. (2017). Theorizing and Measuring Religiosity Across Cultures.
Personality and Social Psychology Bulletin, 43(12), 1724-1736. doi:10.1177/0146167217727732

Cotton, S., McGrady, M. E., \& Rosenthal, S. L. (2010). Measurement of Religiosity/Spirituality in Adolescent Health Outcomes Research: Trends and Recommendations. Journal of Religion and Health, 49(4), 414-444. doi: 10.1007/ s10943-010-9324-0

Flere, S. \& Lavrič, M. (2008). Is intrinsic religious orientation a culturally specific American Protestant concept? The fusion of intrinsic and extrinsic religious orientation among non-Protestants. European Journal of Social Psychology, 38(3), 521-530. doi:10.1002/ejsp.437

Fowler, J. W. (1981). Stages of faith. New York, NY, US: HarperCollins

Fowler, J. W. \& Dell, M. L. (2004). Stages of faith and identity: birth to teens. Child and Adolescent Psychiatric Clinics of North America, 13(1), 17-33. doi:10.1016/s1056-4993(03)00073-7

Fowler, J. W. \& Dell, M. L. (2006). Stages of Faith From Infancy Through Adolescence: Reflections on Three Decades of Faith Development Theory. In E. C. Roehlkepartain, P. E. King, L. Wagener, \& P. L. Benson (Eds.), The handbook of spiritual development in childhood and adolescence (pp. 34-45). Thousand Oaks, CA, US: Sage Publications, Inc. doi: http:// dx.doi.org/10.4135/9781412976657.n3

Gorsuch, R. L. \& McPherson, S. E. (1989). Intrinsic/extrinsic measurement: I/E-revised and single-item scales. Journal for the Scientific Study of Religion, 28(3), 348-354.

Gorsuch, R. L. \& Venable, G. D. (1983). Development of an "Age Universal" I-E Scale. Journal for the Scientific Study of Religion, 22(2), 181-187. doi:10.2307/1385677

Greening, L. \& Stoppelbein, L. (2002). Religiosity, Attributional Style, and Social Support as Psychosocial Buffers for African American and White Adolescents? Perceived Risk for Suicide. Suicide and Life-Threatening Behavior, 32(4), 404-417. doi:10.1521/suli.32.4.404.22333

Gunzler, D. D. \& Morris, N. (2015). A tutorial on structural equation modeling for analysis of overlapping symptoms in co-occurring conditions usingMPlus. Statistics in Medicine, 34(24), 3246-3280. doi:10.1002/sim.6541

Hackney, Ch.H. \& Sanders. G.S. (2003). Religiosity and Mental Health: A Meta-Analysis of Recent Studies. Journal for the Scientific Study of Religion, 42(1), 43-56.

Hardy, S. A., Steelman, M. A., Coyne, S. M., \& Ridge, R. D. (2013). Adolescent religiousness as a protective factor against pornography use. Journal of Applied Developmental Psychology, 34(3), 131-139. doi:10.1016/j.appdev.2012.12.002

Hefetz, A. \& Liberman, G. (2017). The factor analysis procedure for exploration: a short guide with examples / El análisis factorial exploratorio: una guía breve con ejemplos, Cultura y Educación. doi: 10.1080/11356405.2017.1365425

Hernández, R., Fernández, C., \& Baptista, P. (2010). Metodología de la Investigación. México: McGraw - Hill.

Holmes, C. \& Kim-Spoon, J. (2015). Why are Religiousness and Spirituality Associated with Externalizing Psychopathology? A Literature Review. Clinical Child and Family Psychology Review, 19(1), 1-20. doi:10.1007/s10567-015-0199-1

Howard, J. L., Gagné, M., S., A. J., \& Forest, J. (2018). Using Bifactor Exploratory Structural Equation Modeling to Test for 
a Continuum Structure of Motivation. Journal of Management, 44(7), 2638-2664. doi: https://doi.org/10.1177/0149206316645653

Hu, L. \& Bentler, P. M. (1999). Cutoff criteria for fit indexes in covariance structure analysis: Conventional criteria versus new alternatives. Structural Equation Modeling: A Multidisciplinary Journal, 6(1), 1-55. doi:10.1080/10705519909540118

Kim, D. H., Harty, J., Takahashi, L., \& Voisin, D. R. (2017). The Protective Effects of Religious Beliefs on Behavioral Health Factors Among Low Income African American Adolescents in Chicago. Journal of Child and Family Studies, 27(2), 355-364. doi:10.1007/s10826-017-0891-5

Kim, K. H. (2005). The Relation Among Fit Indexes, Power, and Sample Size in Structural Equation Modeling. Structural Equation Modeling: A Multidisciplinary Journal, 12(3), 368-390. doi:10.1207/s15328007sem1203_2

King, P. E. \& Roeser, R. W. (2009). Religion and Spirituality in Adolescent Development. Handbook of Adolescent Psychology. doi:10.1002/9780470479193.adlpsy00101

Koenig, H. G. (2018). Measurement of Religiosity. Religion and Mental Health, 21-48. doi:10.1016/b978-0-12-811282-3.00002-1

Koenig, H. G. (2015). Religion, spirituality, and health: A review and update. Advances in Mind - Body Medicine, 29, 19-26. Retrieved from https://search-proquest-com.ezproxy.biblio.uta. cl/docview/1698024209? accountid $=44468$

Lemos, C. M., Gore, R. J., Puga-Gonzalez, I., \& Shults, F.L. (2019). Dimensionality and factorial invariance of religiosity among Christians and the religiously unaffiliated: A cross-cultural analysis based on the International Social Survey Programme. PLoSONE, 14(5), e0216352. doi: 10.1371/journal.pone.0216352

Li, C-H. (2016). Confirmatory factor analysis with ordinal data: Comparing robust maximum likelihood and diagonally weighted least squares. Behavior Research Methods, 48, 936-949. doi: 10.3758/s13428-015-0619-7

Lloret-Segura, S., Ferreres-Traver, A., Hernández-Baeza, A., \& Tomás-Marco, I. (2014). El análisis factorial exploratorio de los ítems: Una guía práctica, revisada y actualizada [Exploratory Item Factor Analysis: A practical guide revised and updated]. Anales de Psicología, 30(3), 1151-1169.

Maltby, J. (1997). An amended version of a religious orientation measure: Some data on reliability and validity among English and Northern Irish schoolchildren. The Irish Journal of Psychology, 18(4), 448-450. doi:10.1080/03033910.1997.101055816

Maltby, J. (1999) The internal structure of a derived, revised, and amended measure of the Religious Orientation Scale: The 'Age-Universal' I-E scale 12. Social Behaviour and Personality, 27, 407-412

Marsh, H. W., Morin A.J., Parker P. D., \& Kaur, G. (2014). Exploratory structural equation modeling: an integration of the best features of exploratory and confirmatory factor analysis. Annual Review of Clinical Psychology, 10, 85-110. doi: 10.1146/ annurev-clinpsy-032813-153700.

Marsh, H. W., Nagengast, B., \& Morin, A. J. S. (2013). Measurement invariance of big-five factors over the life span: ESEM tests of gender, age, plasticity, maturity, and la dolce vita effects. Developmental Psychology, 49(6), 1194-1218. doi: http://dx.doi.org/10.1037/a0026913
McDonald, R. P. \& Ho, M.-H. R. (2002). Principles and practice in reporting structural equation analyses. Psychological Methods, 7(1), 64-82. doi: http://dx.doi.org/10.1037/1082-989X.7.1.64

Morales, P. (2007). Estadística aplicada a las Ciencias Sociales. La fiabilidad de los tests y escalas. Universidad Pontificia Comillas, Madrid. Recuperado de https://matcris5.files.wordpress.com/2014/04/fiabilidad-tests-y-escalas-morales-2007.pdf

Neymotin F. \& Downing-Matibag T.M. (2013). Religiosity and adolescents' involvement with both drugs and sex. Journal of Religion \& Health, 52(2), 550-569. doi: 10.1007/ s10943-011-9507-3.

Nunnally, J. C. (1987). Teoría psicométrica. México. Trillas.

Pössel, P., Martin, N. C., Garber, J., Banister, A. W., Pickering, N. K., \& Hautzinger, M. (2011). Bidirectional relations of religious orientation and depressive symptoms in adolescents: A short-term longitudinal study. Psychology of Religion and Spirituality, 3(1), 24-38. doi:10.1037/a0019125

Putnick, D. L. \& Bornstein, M. H. (2016). Measurement invariance conventions and reporting: The state of the art and future directions for psychological research. Developmental Review, 41, 71-90. doi:10.1016/j.dr.2016.06.004

Rew, L. \& Wong, Y. J., (2006). A systematic review of associations among religiosity/spirituality and adolescent health attitudes and behaviors. Journal of Adolescent Health, 38(4), 433-442. doi: https://doi.org/10.1016/j.jadohealth.2005.02.004

Rivera-Ledesma, A., Zavala-Jiménez, S., \& Montero-López, M. (2016). Validación de la Age Universal I-E Scale en sujetos mexicanos. Universitas Psychologica, 15(4). doi: http://dx.doi. org/10.1114/Javeriana.upsy15-4.vaus

Ryu, E. (2011) Effects of skewness and kurtosis on normal-theory based maximum likelihood test statistic in multilevel structural equation modeling. Behavior Research Methods, 43(4), 1066-1074. doi: 10.3758/s13428-011-0115-7

Satorra, A. (2000). Scaled and adjusted restricted tests in multi-sample analysis of moment structures. In R. D. H. Heijmans, D. S. G. Pollock, \& A. Satorra (Eds.), Innovations in multivariate statistical analysis. A festschrift for Heinz Neudecker (pp. 233-247). London: Kluwer Academic.

Schreiber, J. C. \& Culbertson, M. J. (2014). Religious socialization of youth involved in child welfare. Child Abuse \& Neglect, 38(7), 1208-1220. doi: http://dx.doi.org/10.1016/j.chiabu.2014.03.021

Sharma, S., Mustanski, B., Dick, D., Bolland, J., \& Kertes, D.A. (2019). Protective Factors Buffer Life Stress and Behavioral Health Outcomes among High-Risk Youth. Journal of Abnormal Child Psychology, 47, 1289. doi: https://doi.org/10.1007/ s10802-019-00515-8

Simkin, H. \& Etchezahar, E. (2013). Las orientaciones religiosas extrínseca e intrínseca: validación de la "Age Universal" I-E Scale en el contexto argentino. Psykhe, 22(1), 97-106.

Xing, C. \& Hall, J. A. (2015). Confirmatory Factor Analysis and Measurement Invariance Testing with Ordinal Data: An Application in Revising the Flirting Styles Inventory. Communication Methods and Measures, 9(3), 123-151. doi:1 0.1080/19312458.2015.1061651

Zwingmann, C., Klein, C., \& Büssing, A. (2011). Measuring Religiosity/Spirituality: Theoretical Differentiations and Categorization of Instruments. Religions, 2(3), 345-357. doi:10.3390/rel2030345 